Research Article

\title{
Comparison of Effects of Radiofrequency Ablation of Liver Cancer Guided by CT Images Based on Deep Learning Algorithm
}

\author{
Kai Huang $\mathbb{D}^{1},{ }^{1}$ Tongqing Zhang $\mathbb{D}^{2}{ }^{2}$ Zhiliang $H e \mathbb{D}^{3},{ }^{3}$ and Fengxia Wang $\mathbb{D}^{1}$ \\ ${ }^{1}$ Department of Infectious Diseases, Dezhou People's Hospital, Dezhou 253014, China \\ ${ }^{2}$ Department of Laboratory, Dezhou Municipal Hospital Branch, Dezhou 253016, China \\ ${ }^{3}$ Department of Medical Imaging, Dezhou Municipal Hospital Branch, Dezhou 253016, China
}

Correspondence should be addressed to Fengxia Wang; 20150304046@m.scnu.edu.cn

Received 19 June 2021; Accepted 2 August 2021; Published 10 September 2021

Academic Editor: Gustavo Ramirez

Copyright (c) 2021 Kai Huang et al. This is an open access article distributed under the Creative Commons Attribution License, which permits unrestricted use, distribution, and reproduction in any medium, provided the original work is properly cited.

\begin{abstract}
Objective. To investigate the paper radiofrequency ablation (RFA) CT-guided feasibility of hepatocellular carcinoma (primary liver cancer) treatment, safety, and clinical efficacy of the use of deep learning algorithms. Method. A total of 47 cases of primary liver cancer patients were included: 21 cases of CT-guided liver lesions in line with RFA (C-CT group) and, in the same period, 26 cases of spiral CT-guided liver lesions in line with RFA (S-CT group). Two groups of patients were recorded immediately after the total operation time and ablation time, the acceptable radiation dose was observed in the incidence of postoperative complications of $7 \mathrm{~d}$, and the postoperative hospital stay was recorded to evaluate the efficacy of the treatment of lesions in 1,3 , and 6 months after RFA. Results. All 47 patients were successful; two technical success rates were $100 \%$. There was a significant difference $(P<0.05)$ in the total operation time groups, ablation time, and acceptable radiation dose. And there was no significant difference $(P<0.05)$ in postoperative complications of $7 \mathrm{~d}$ groups, postoperative hospital stay, and local disease control. There was a significant difference $(P<0.05)$ in the S-CT group, seven ablation residual or recurrent lesions during the follow-up ratio of $26.9 \%$, and C-CT groups compared with only $14.3 \%$. Conclusion. CT-guided RFA treatment of primary liver cancer patients is safe, effective, and superior to the conventional spiral CT-guided ablation lesion site-specific terms.
\end{abstract}

\section{Introduction}

Currently, surgical resection of primary liver cancer and liver transplantation is the preferred treatment, but more than $80 \%$ of patients at the time of diagnosis have lost the opportunity for surgery, and the 5 -year recurrence rate is about $40 \%$ to $70 \%$. For unresectable primary liver cancer and postoperative recurrence, treatment can be through a variety of interventional transcatheter arterial chemoembolization and physical ablation, etc., and has been widely used in clinical practice. Radiofrequency ablation (RFA) as a minimally invasive method for curative treatment in the field of interventional tumors has been widely carried out. Substantial research and clinical guidelines state that, in patients with small diameter $<3 \mathrm{~cm}$ liver cancer, there is no significant difference in the overall survival of patients after RFA and relapse-free survival aspects of surgical resection.
However, even if the above two image guidance methods are used in combination, there are still some inevitable limitations and defects in the actual operation process, which also weakens the therapeutic effect of RFA to a certain extent. As a relatively new imaging device, CT is usually integrated into an angiography machine. Compared with traditional spiral CT guidance, RFA treatment under CT guidance has unique technical advantages. Through the rotation, the surgeon can obtain the stereoscopic perspective image of the patient in real time; at the same time, CT can also postprocess the acquired perspective image to obtain the tomographic image of the patient. In recent years, CTguided RFA has received more and more attention and application, and our center has gradually carried out RFA treatment under CT guidance since 2016. This article aims to retrospectively analyze the clinical data and follow-up data of patients treated with RFA under CT guidance in this 
center and compare it with the data of patients under conventional spiral CT-guided RFA treatment in order to evaluate the new image-guided technology in the treatment safety and effectiveness in primary liver cancer [1].

\section{Information and Methods}

2.1. General Information. From September 2016 to October 2017, 21 cases of primary liver cancer patients under CTguided RFA treatment (C-CT group) were collected in our center, and 26 cases of primary liver cancer patients underwent RFA treatment under spiral CT guidance during the same period (S -CT group). In the C-CT group, there were 16 males and 5 females, aged 29 to 76 years, with an average of $55.7 \pm 11.8$ years; tumor size is $3.4 \pm 1.9 \mathrm{~cm}$; Child-Pugh grades $\mathrm{A}$ and $\mathrm{B}$ were 20 and 1 cases, respectively; BCLC stages $\mathrm{A}$ and $\mathrm{B}$ were 9 and 12 cases, respectively; ECOG scores were 0 and 1 for 16 and 5 cases, respectively; yes and no vascular invasion were 0 and 21 cases, respectively; yes and no extrahepatic metastasis were 4 and 17 cases, respectively. In the S-CT group, there were 20 males and 6 females, aged 41-86 years, with an average of $65.2 \pm 10.4$ years; tumor size is $2.7 \pm 1.2 \mathrm{~cm}$; Child-Pugh grades A and B were 24 and 2 cases, respectively; BCLC stages A and B were 11 and 15 cases, respectively; ECOG scores were 0 and 1 for 18 and 8 cases, respectively; yes and no vascular invasion were 0 and 26 cases, respectively; yes and no extrahepatic metastasis were 1 and 25 cases, respectively. Except for the slightly younger age of the patients in the C-CT group, there was no significant difference in the general data between the two groups, and they were comparable [2]. The RFA treatment of all 47 lesions was performed by the same team using a unified angiography machine (France) and radiofrequency ablation equipment (1500X radio frequency generator and Starburst radiofrequency needle, RITA, USA). In terms of clinical data and follow-up data, all patients completed imaging and related laboratory examinations before surgery and completed the enhanced CT or MR examination follow-up 1, 3, and 6 months after surgery.

2.2. Equipment. The image guidance equipment uses Dehisced 8-row spiral CT. The RFA system is a cold-circulation radiofrequency ablation system of Guangzhou Zhuhai Hejia Medical Equipment Co., Ltd. Radiofrequency ablation needle: HG-20 controllable cold pole needle (monopolar needle, radiofrequency range $2.5 \sim 3.5 \mathrm{~cm}$ ).

2.3. Method. All patients underwent TACE ( 1 to 2 times), and CT was reviewed 4 to 6 weeks later. Those with recurrence or remnants were considered for CT-guided RFA. Among them, 2 patients underwent RFA with the direct puncture of liver surface tumor lesions. APF was regularly reviewed after RFA, liver function, and CT or MRI to evaluate the efficacy.

2.3.1. TACE. After performing hepatic arteriography with a $4 \mathrm{~F}$ or $5 \mathrm{FRH}$ catheter, we superselect the tumor-providing blood vessels. If the tumor's blood-supplying artery is tortuous and slender, we use a microcatheter to superselect the tumor-feeding blood vessels. First, the chemotherapy drug 5-Fu500 $1000 \mathrm{mg}$ is routinely injected for local infusion chemotherapy. The dosage of the drug is determined according to the patient's white blood cells and platelet counts and liver function. Then, we use $5-20 \mathrm{ml}$ of ultraliquid iodide oil and 20-40 mg of pirarubicin or 10-20 mg of hydroxycamptothecin (selected when the cardiac function is abnormal) to make a suspension for perfusion embolization.

2.3.2. Under General Anesthesia, CT-Guided RFA. All patients underwent general anesthesia with endotracheal intubation vein. Induced anesthesia medication is as follows: injection of diazepam of $1 \sim 2 \mathrm{mg} / \mathrm{kg}$, midazolam of $0.1 \mathrm{mg} /$ $\mathrm{kg}$, fentanyl of $1 \sim 3 \mu \mathrm{g} / \mathrm{kg}$, and muscle relaxation drug rocuronium of $0.6 \mathrm{mg} / \mathrm{kg}$. Drugs for maintaining anesthesia are as follows: atracurium cis benzenesulfonate of $0.2 \mathrm{mg} / \mathrm{kg}$, propofol of 1 to $2 \mathrm{mg} / \mathrm{kg}$, administered using a target concentration-controlled infusion mode, and plasma drug concentration of 2.5 to $4.0 \mu \mathrm{g} / \mathrm{ml}$. There should be intraoperative dynamic monitoring of vital signs such as heart rate, blood pressure, and $\mathrm{SaO}_{2}$.

The patient was placed in a supine position and positioned on the corresponding body surface area according to the location of the tumor. The HI Speed 8-row spiral CT scanned the liver to confirm the location of the tumor and determine the puncture direction, angle, and needle penetration depth. We select the appropriate puncture path and determine the puncture point on the CT image in combination with the positioning scale. After disinfecting the towel, we cut a $0.5 \mathrm{~cm}$ incision at the puncture site, the puncture of the RF electrode needle gradually entered the RF target site, and the CT scan determined that the needle tip of the RF electrode was located in the ablation target area for RF ablation treatment. We choose the temperature and time of treatment according to the location and size of the tumor. The commonly used radio frequency conditions are the temperature of $70^{\circ} \mathrm{C} \sim 90^{\circ} \mathrm{C}$ and time of $12 \sim 18 \mathrm{~min}$. When the tumor is large, single or multiple multipoint radiofrequency treatment is feasible. After the treatment, the needle channel radiofrequency hemostasis, CT scan was done to see the treatment effect and to determine whether there are complications such as subhepatic hemorrhage and pneumothorax. Heart rate, blood pressure, and $\mathrm{SaO}_{2}$ vital signs were continuously measured 24 hours after surgery (Figures 1 and 2).

Combined with general anesthesia surgery to expose the liver surface tumors under direct vision for RFA treatment, under general anesthesia, through the right oblique incision of the right costal margin, we free the liver to fully reveal the tumor, select the puncture point and the direction and angle of needle insertion according to the tumor site and size, and directly puncture the RF electrode needle to the center of the tumor under direct vision. After confirming that it is correct, we start RF sequential treatment (same as above). During the entire RFA treatment, we pay attention to observe the color change of the tumor tissue, which generally changes from dark red to gray and white and further to black or carbonization [3]. 

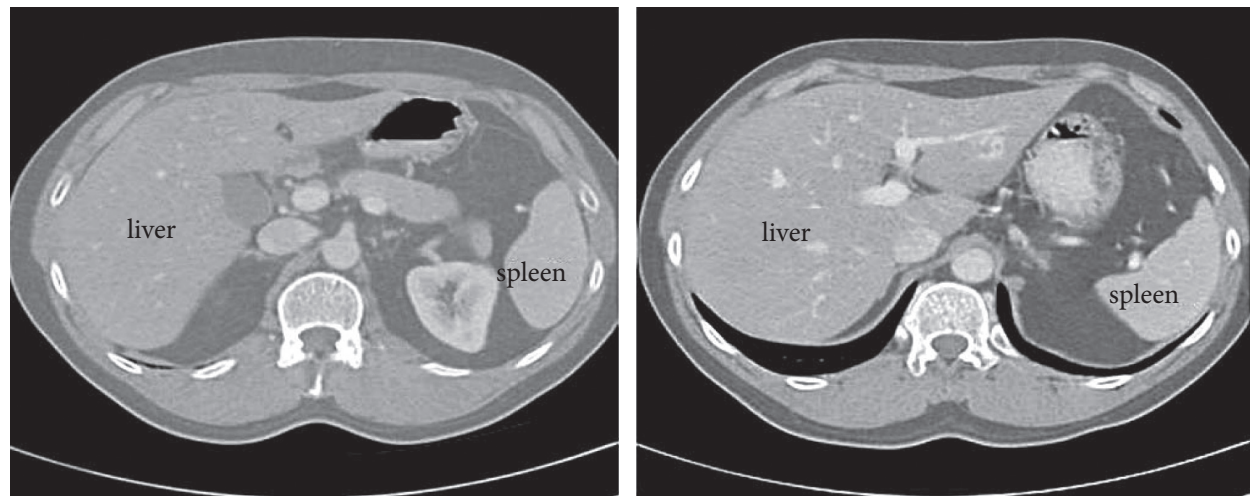

FIGURE 1: Marking of the patient's respiratory baseline during CT-guided RFA.
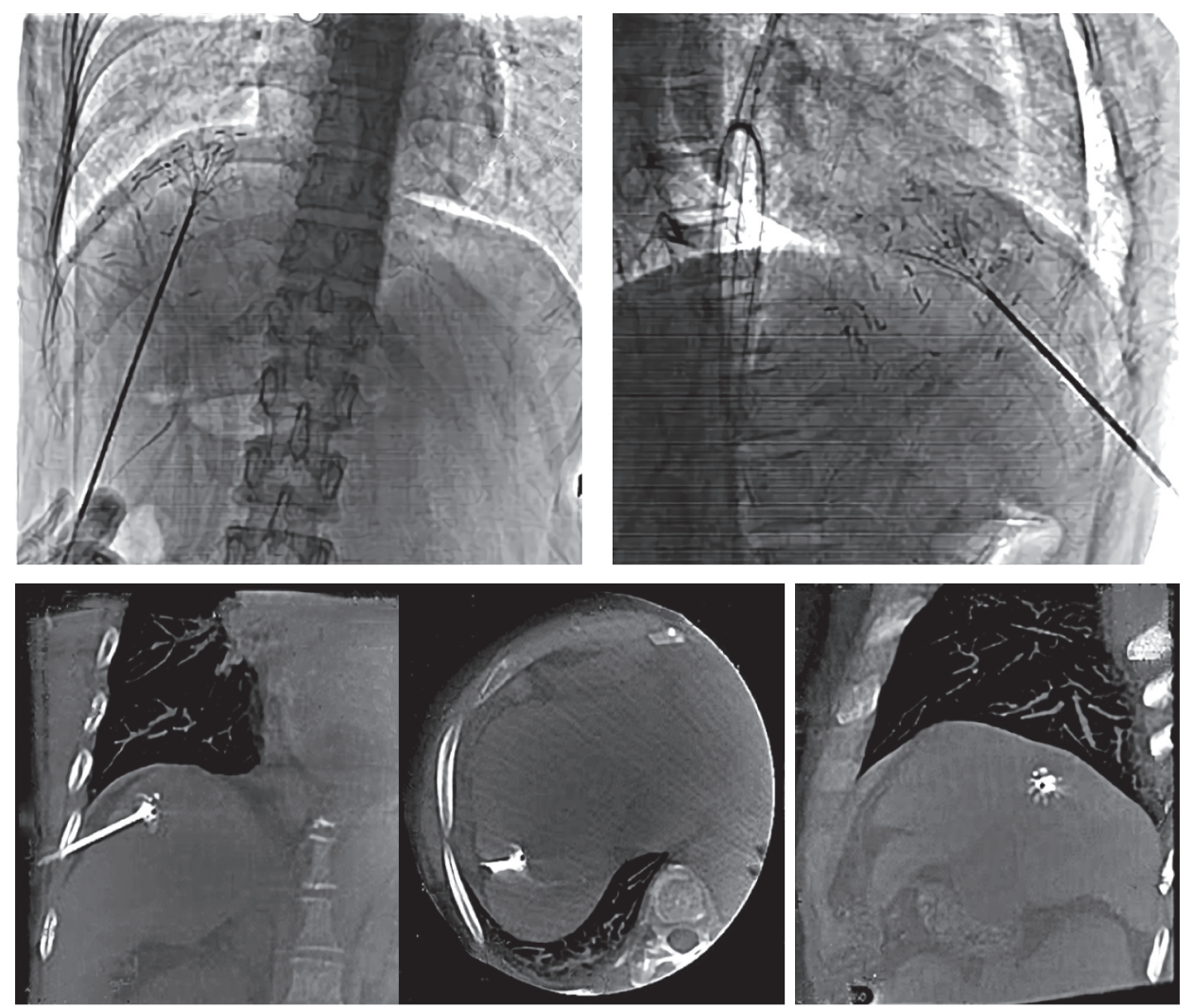

Figure 2: Simplified perspective view of the operation to adjust the angle of the needle in real time.

After judging the tumor changes, they were reviewed every 3 months and every 6 months after 1 year and were followed up for up to 3 years. The short-term curative effect of a tumor is evaluated by imaging according to the WHO solid tumor evaluation standard. The evaluation criteria of efficacy are as follows: (1) complete remission (CR): the tumor completely disappears, and imaging examination cannot show the tumor; (2) partial remission (PR): the tumor shrinks, and the product of the maximum diameter of the tumor is reduced by $\geq 50 \%$ compared with before treatment; (3) stability (SD): the product of the largest tumor diameter decreases by $<25 \%$ or increases by $<25 \%$ compared to that before treatment; (4) progress (PD): the product of the largest tumor diameter increases by $\geq 25 \%$ or new lesions appear before treatment.

2.4. The Principle of Deep Learning Algorithm Processing CT Image. One-dimensional signal: $f(t) \in L^{2}(R)$, standard orthonormal basis expansion in subspace $V_{m+1}$, and equation (1) can be obtained.

$$
f(t)=\sum_{n} c_{m+1, n} \phi_{m+1, n}
$$

In the formula, $c_{m+1}=\left\langle f, \phi_{m+1}\right\rangle . f(t)$ can be described as 


$$
f(t)=\sum_{k} c_{m k} \phi_{m k}(t)+\sum_{k} d_{m k} \varphi_{m k(t)} .
$$

In the formula, $\sum_{k} c_{m k} \phi_{m k \gamma}(t)$ and $\sum_{k} d_{m k} \varphi_{m k(t)}$ represent the low-frequency part and high-frequency part of the onedimensional signal, respectively.

$$
\begin{aligned}
c_{m k} & =\sum_{n} h o_{(n-2 k)}(t) c_{m+1, n}, \\
d_{m k} & =\sum_{n} h_{1(n-2 k)}(t) d_{m+1, n} .
\end{aligned}
$$

In the formula, $h_{0(n-2 k)}=(1 / \sqrt{2}) \int_{\phi}((\tau / 2)-k) \phi$ $(t-n) \mathrm{d} t, h_{1(n-2 k)}=(1 / \sqrt{2}) \int \Psi((\tau / 2)-k) \phi(t-n) \mathrm{d} t$.

Image $f(x, y)$ is a two-dimensional signal, so it is necessary to establish a two-dimensional scale function, specifically formula (4).

$$
\phi(x, y)=\phi(x) \phi(y) .
$$

For image $f(x, y)$, we use $\phi(x)$ and $\Psi(x)$ analysis in the $x$ direction to obtain the approximation part and the detail part and then perform the same operation in the $y$ direction to obtain the low-frequency subimage $A_{1} f(x, y)$, horizontal detail subimage $D_{1}^{(1)} f(x, y)$, vertical detail subimage $D_{1}^{(2)} f(x, y)$, and diagonal detail subimage $D_{1}^{(3)} f(x, y)$.

A careful analysis of Figure 3 shows that the low-frequency subimage preserves the main features of the original image, so it is used as the image classification feature, but the original image classification feature dimension is quite high. In order to reduce the image dimension and improve the image classification efficiency, the feature vector of the original image is subjected to principal component analysis processing, and the principal component of the feature vector of the original image is selected [4].

2.5. Statistical Processing. All data statistics are carried out using the SPSS13.0 software package, counting data using chi-square test, and measurement data using $t$-test. $P<0.05$ was considered statistically significant.

\section{Results}

3.1. General Situation. All 47 patients had successful surgery; two technical success rates were $100 \%$. The total C-CT operation time was $90.5 \pm 33.8 \mathrm{~min}$; ablation time was $29.3 \pm 18.2 \mathrm{~min}$, receiving radiation dose of $243.6 \pm 192.6$ mGy; total S-CToperation time was $65.8 \pm 15.1 \mathrm{~min}$; ablation time was $20.5 \pm 11.6 \mathrm{~min}$, receiving a dose of radiation of $2317 \pm 1292 \mathrm{mGy}$. Concerning the total operation time groups, ablation time, and comparison of reception radiation dose aspect, the difference was significant $(t=-3.342 \sim 7.284, P<0.05)$. Concerning the C-CT postoperative hospital stay group $(4.0 \pm 1.0) d$ and S-CT group $(3.7 \pm 0.9) d$, there was no significant difference between the two groups $(t=-977, P>0.05)$ (Figures $4-6)$.

3.2. Efficacy Analysis. AFP: 3 cases of liver metastases in this group were normal before and after AFP treatment, and the carcinoembryonic antigen (CEA) increased significantly. The remaining 38 patients were AFP elevated, ranging from 16.4 to $1378 \mathrm{U} / \mathrm{ml}$. In the elevated AFP type, the preoperative AFP and postoperative AFP were $136.6 \pm 56.7 \mathrm{U} / \mathrm{ml}$ and $19.2 \pm 10.8 \mathrm{U} / \mathrm{ml}$, respectively, and the difference was statistically significant $(t=18.61, P<0.01)$. In one patient, AFP decreased to normal after the operation. Efficacy evaluation: forty-one patients with 48 tumors underwent 43 RFA treatments. All patients were followed up for 2 to 36 months, with a median follow-up period of 16 months, and the follow-up rate was $100 \% .12$ cases were CR and 16 cases were PR. Three cases were SD. The CT scan of CR patients after RFA showed that the CT value of the treatment area generally decreased. The range of the lesion was slightly larger than that before treatment, and the boundary was unclear. With time, the edge of the lesion gradually became clear. The center density of the treatment area of 5 patients was higher than the surrounding density. In patients with $\mathrm{CR}$, the enhanced CT necrosis area is not enhanced at all stages, showing a low density, and the tumor morphology and margins are clearly displayed. The residual tumor tissue of PR patients is not strengthened in the center of the arterial phase, but there are nodular or strip-shaped enhancements around it. Survival rate: 41 patients were followed up for an average of 16 months, and the overall survival rates at 1 and 2 years were $87.5 \%$ and $74.4 \%$, respectively [5].

3.3. Complications. There were no serious complications in this group of patients. In some cases, postoperative hepatic subcapsular hemorrhage and pain at the puncture site were relieved after symptomatic treatment. There were no liver abscess, biliary peritonitis, abdominal organ damage, hemothorax, puncture tract metastasis, liver failure, and death.

\section{Discussion}

TACE has been used in the treatment of liver cancer at home and abroad for a long time, and its efficacy has also been affirmed. However, it has been found in a large number of clinical practices that TACE does not completely cause tumor necrosis. The complete tumor necrosis rate is only about $20 \%$, which may be related to collateral circulation, multiarterial blood supply, and partial blood supply around the tumor from the portal vein, and incomplete embolization other factors are related; in addition, tumors with poor blood supply have worse efficacy, so local recurrence and progression of tumors will occur after TACE alone, and patients with liver cancer are often accompanied by hepatitis and cirrhosis background. Repeated TACE will increase cirrhosis, which affects the quality of life of patients and causes some patients to die not from liver cancer but from severe cirrhosis and its complications.

RFA is a percutaneous puncture guided by CT or ultrasound imaging technology, which inserts radiofrequency electrodes directly into tumor tissue, emits high-frequency radiofrequency waves, stimulates tissue cells to generate 

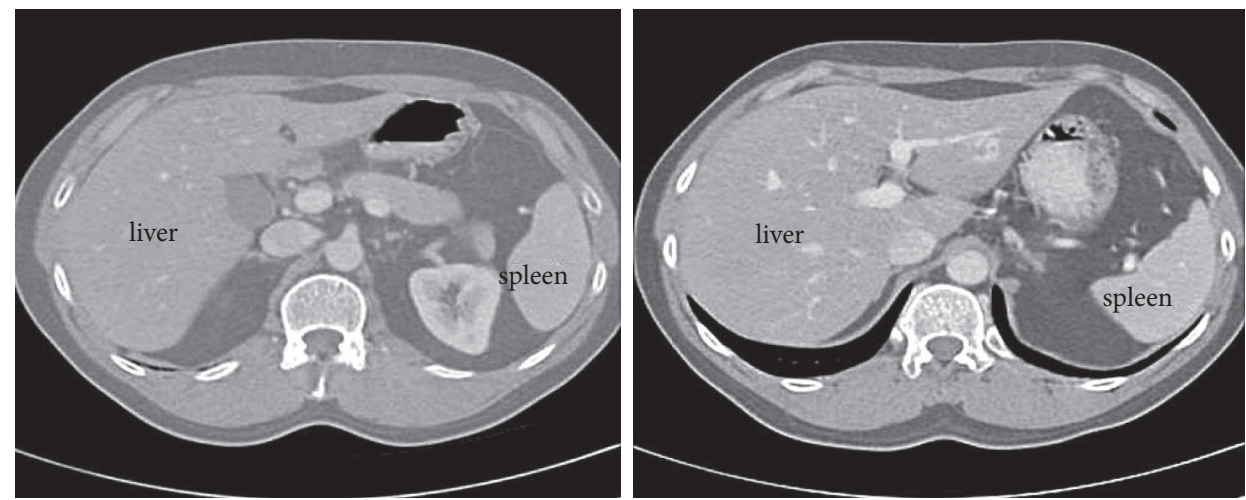

Figure 3: Two-dimensional wavelet transform of the image.

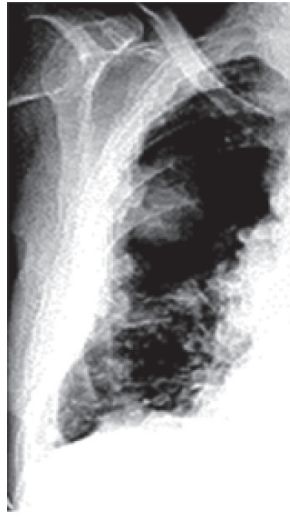

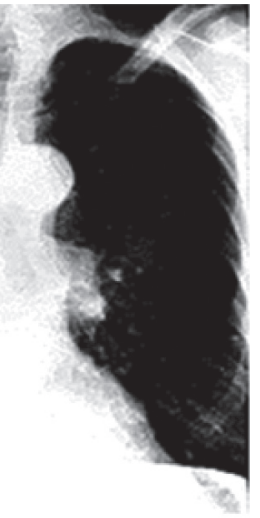

(a)

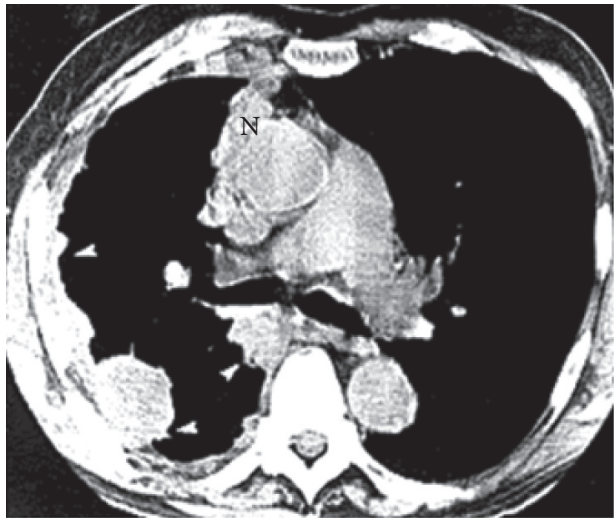

(b)

FIGURE 4: CT appearance after needle extraction (see lesion necrosis and gasification).
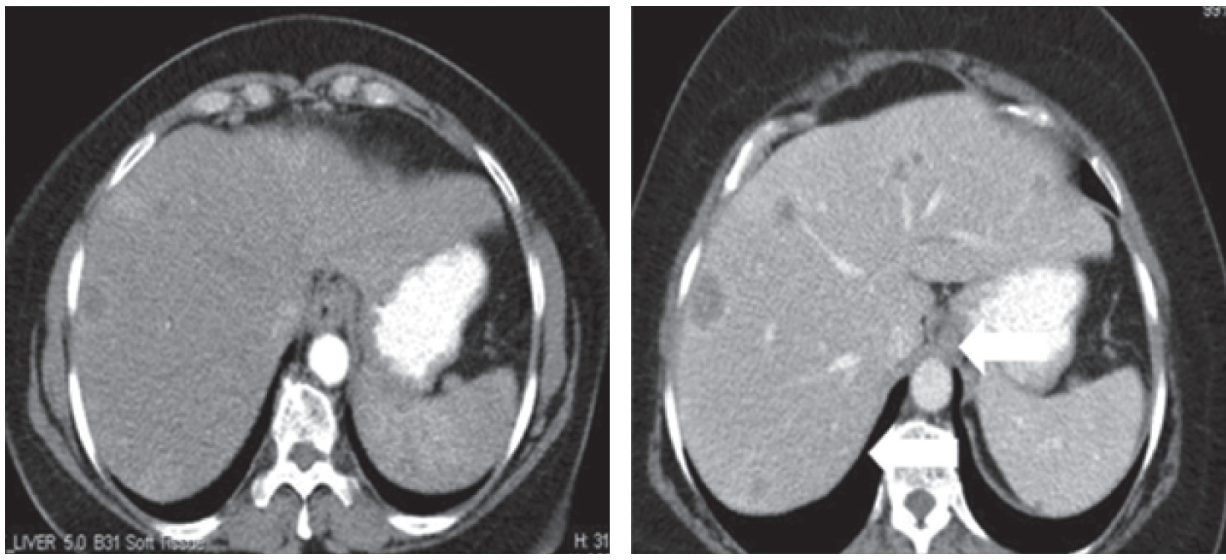

FIGURE 5: CT lipiodol review after TACE failed to completely precipitate the lesion.

high-speed ion oscillation and friction, and generates thermal energy. The local temperature reaching $70^{\circ} \mathrm{C} \sim$ $120^{\circ} \mathrm{C}$ can cause tissue coagulation and necrosis, an irreversible degeneration of mitochondria, lysosomes, proteins, and DNA in cells, cut off the blood supply of tumors, and prevent tumor metastasis. RFA is equivalent to removing the tumor from the function and can even achieve an effect similar to resection. RFA can repeat additional treatment for recurrent lesions to consolidate and improve the efficacy. It is reported that thermal ablation therapy still has an immunological response; that is, it can activate and enhance the host's antitumor immune function, especially the cellular immune function of patients' peripheral blood, and local treatment area has been significantly enhanced, which may 


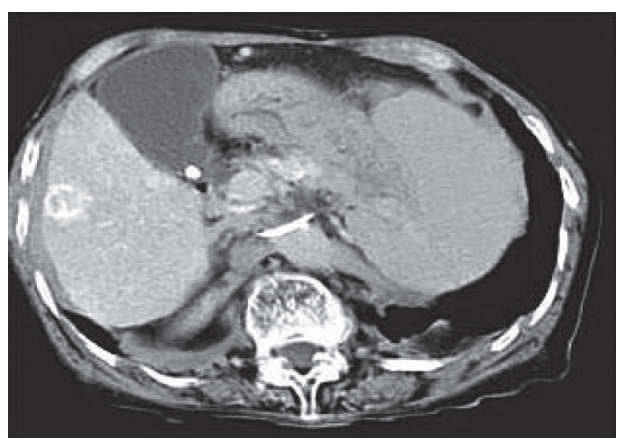

(a)

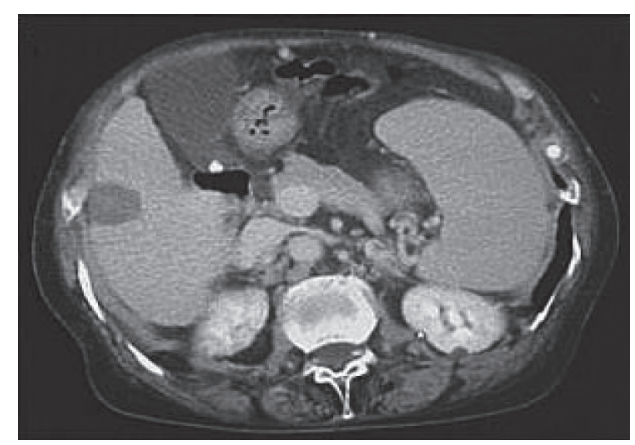

(b)

Figure 6: After RFA, there is no enhancement around the CT lesion.

be an important factor for the good efficacy of some patients. The combination of TACE and RFA in the treatment of primary liver cancer has achieved good results. The postoperative AFP is significantly lower than that before the operation. Of the 28 patients with elevated AFP, 4 patients had normal postoperative AFP. 12 cases were CR (38.7\%), 16 cases were PR (51.6\%), and 3 cases were SD (9.7\%). (1) After TACE, RFA treatment can block the blood supply of the tumor, thereby eliminating the heat deposition effect, increasing the local temperature of the tumor, and ultimately improving the therapeutic effect of RFA and expanding the scope of RFA treatment; (2) after TACE, iodine deposits can play a guiding role to determine the scope of RFA treatment and reduce the chance of tumor puncture bleeding and blood channel metastasis during RFA treatment.

In this group of cases, 2 cases are located near the gallbladder, gastrointestinal tract, kidney, and other special parts of the liver, such lesions are difficult to puncture due to needle penetration, thermal ablation range is easy to damage the surrounding structures, and RFA thermal ablation energy is easily susceptible to blood flow or bile is taken away, which limits the application of RFA. Therefore, it must be accurately positioned before surgery, and the patient should breathe uniformly every time to avoid a puncture through the lung tissue and repeated puncture. During the puncture, we try to pass through normal liver tissue to avoid direct puncture of the lesion; the plain scan of liver metastases is of equal density, which is not easy to display clear and accurate positioning. In order to ensure patient safety and interventional efficacy, the author performs enhanced scans before puncture, according to puncture treatment after accurate positioning of the lesion enhancement range and location [6].

In 1998, Livraghi et al. proposed the hypothesis of the "oven effect" in the treatment of liver cancer in the context of radiofrequency cirrhosis; that is, the cirrhotic liver tissue around the tumor played an adiabatic role, making the heattransferred tumor tissue more prone to necrosis. On the other hand, the outer edge of liver metastasis tumors infiltrates the surrounding liver parenchyma, and the probability of local recurrence of tumors due to omissions is relatively high. Therefore, when ablating metastatic liver cancer, more radical methods should be adopted, such as expanding the safe ablation range, to reduce the local recurrence rate of tumors. In addition, patients with liver metastatic cancer often do not have cirrhosis, so appropriately expanding the ablation range will not cause serious complications such as liver failure.

Due to the high temperature of the radiofrequency ablation focus (above $70^{\circ} \mathrm{C}$ ) during the treatment, it inevitably caused a series of adverse reactions such as chest pain, high fever, sweating, irritability, continuous increase in blood pressure, fast heart rate, and even nausea and vomiting, which make patients extremely painful, even more, so because the reaction is too strong and the pain is unbearable, the treatment is terminated, which affects the treatment to a certain extent. RFA under general anesthesia can effectively eliminate or alleviate the suffering of patients, reduce the occurrence of complications, facilitate surgical operation, and be easily accepted by patients. In the early stage of this group, only one patient was treated with local anesthesia. The patient clearly felt right upper abdominal pain and irritability during the operation. Later, the author used radiofrequency treatment under general anesthesia, which obviously eliminated the discomfort during the operation.

Due to the special location of the liver, some tumors are located at the top of the diaphragm or next to the diaphragm, and some are close to the hilum. Percutaneous puncture is very likely to cause lung injury, diaphragm injury, or large blood vessel damage. It is sometimes very difficult to reveal with the help of laparoscopy. For these patients, Surgery combined with RFA has an irreplaceable advantage; it can provide more accurate positioning or even find additional lesions, such as left liver cancer combined with small right liver metastases and feasible left hepatectomy combined with right liver small liver cancer RFA treatment. It is revealed by surgery that the tumor can be exposed to the visual field and operated under direct vision, which increases safety and can also deal with other problems that need to be solved by surgery, such as simultaneous cholecystectomy and pericardial vascular dissection. It provides a safe and effective treatment for some liver cancer patients.

During the follow-up period, although there was no statistical difference between the ORR and DCR of the two guided methods to ablate the lesion, a careful analysis of the data showed that it was easy to see that, over time, the S-CT 
group continued to have good local control (i.e., efficacy evaluation CR). Local recurrence or new lesions appeared around the lesion, but the C-CT group did not have the above phenomenon. Throughout the follow-up period, 7 cases of ablation lesions in the S-CT group remained or recurred during the follow-up process, and the proportion was $26.9 \%$, while in the C-CT group, this proportion was only $14.3 \%$. Also, to a certain extent, the C-arm CT-guided RFA ablation of the lesion is more complete, the lesion is more thoroughly controlled, and the effect is more stable. For the patients who were not evaluated as $\mathrm{CR}$ in the two groups during the followup period, the treatment was as follows. In the C-CT group, 2 patients had multiple new lesions in the liver at the 1-month follow-up postoperatively. The ablation lesions were supplemented and ablated, and only TACE was performed to control the intrahepatic lesions, but the efficacy was not good, and the intrahepatic lesions continued to progress. In the S-CT group, 1 patient was evaluated for PR at 1 month of follow-up, and the follow-up was selected because of the small residual disease and the poor liver function of the patient. The evaluation was PR at 3 months. At 3 months after surgery, in addition to the above $1 \mathrm{PR}$ lesion, 3 lesions recurred. Among them, a large number of new lesions appeared in the liver of the lesion evaluated as $\mathrm{SD}$, and the opportunity for further treatment has been lost; 2 of the 3 PR lesions were subjected to supplemental ablation and 1 was treated with supplemental TACE, but none of them were evaluated at 6 months and meet CR standards [7].

\section{Conclusion}

In summary, RFA has been widely used in the clinic as a minimally invasive, short course of treatment, with fast curative effect, less complications, and less liver function damage. It has gradually become another important method for nonsurgical treatment of liver cancer. RFA treatment after TACE can kill the remaining viable cancer cells. The two methods complement each other and make tumor tissue necrosis more thorough. TACE combined with CT-guided RFA is effective in the treatment of primary liver cancer in the near future, with a high rate of complete tumor necrosis and safe operation. It is a minimally invasive treatment worthy of promotion.

\section{Data Availability}

The data used to support the findings of this study are available from the corresponding author upon request.

\section{Conflicts of Interest}

The authors declare no conflicts of interest.

\section{References}

[1] A. Jacobs, "Radiofrequency ablation for liver cancer," Radiologic Technology, vol. 86, no. 6, p. 645, 2015.

[2] $\mathrm{S}$. Wu, "A traffic motion object extraction algorithm," International Journal of Bifurcation and Chaos, vol. 25, no. 14, Article ID 1540039, 2015.
[3] R.-x. Chen, Y.-h. Gan, N.-l. Ge, Y. Chen et al., Comparison of transarterial chemoembolization with radiofrequency ablation for unresectable barcelona clinic liver cancer stage 0/a hepatocellular carcinoma: a propensity score matching," Journal of Gastroenterology and Hepatology, vol. 31, no. 2, pp. 442-449, 2016.

[4] Y. Makino, Y. Imai, T. Igura, S. Kogita et al., Feasibility of extracted-overlay fusion imaging for intraoperative treatment evaluation of radiofrequency ablation for hepatocellular carcinoma," Liver Cancer, vol. 5, no. 4, pp. 269-279, 2016.

[5] S. Wu, "Internet public informatioan text data mining and intelligence influence analysis for user intent understanding," Journal of Intelligent and Fuzzy Systems, vol. 38, no. 1, pp. 487-494, 2020.

[6] S. Sheng, B. Sun, J. Zheng, X. Cui, S. Cui, and C. Yuan, "Transcatheter hepatic arterial chemoembolization combined with ct guided radiofrequency ablation for treatment of primary liver cancer in caudate lobe," Chinese Journal of Interventional Imaging and Therapy, vol. 14, no. 7, pp. 391-395, 2017.

[7] S. Wu, J. Liu, and L. Liu, "Modeling method of internet public information data mining based on probabilistic topic model," The Journal of Supercomputing, vol. 75, no. 9, pp. 5882-5897, 2019. 